\title{
No strong or consistent association between early childhood caries and pacifier use
}

\author{
Does the use of a pacifier increase the risk of early childhood caries?
}

Peressini S. Pacifier use and early childhood caries: an evidence-
based study of the literature. J Can Dent Assoc 2003; 69:16-19

Data sources Pub Med (1966-2003), Pre-MEDLINE and MEDLINE (1966-2003), the Social Sciences Citation Index, Full Search (19932003), Healthstar (January 1975-June 2001) and the Science Citation Index Expanded (1993-2003) were sources. Searches were limited to studies of human subjects published in the English language. Reference lists of included articles and of textbooks were also searched.

Study selection Studies were included if they reported primary research and examined early childhood caries (ECC) specifically, and not dental caries of the whole primary dentition. Selection required that they reported on pacifier use (prolonged or short-term and with or without sweetening) as a separate potential risk factor for ECC and that odds ratios were reported or could be calculated from the findings presented. Randomised controlled trials, cohort, case-control or crosssectional study designs were included.

Results Of 74 identified studies, eight met the inclusion criteria. These were methodologically inconsistent in terms of definitions of ECC, diagnostic criteria for identifying carious lesions, dental examination procedures, interviewing methods, and descriptions of pacifier use. Six studies did not control for confounding variables, and the conclusions they generated were inconsistent. The evidence from the other two studies, which did control for confounding factors, presented slightly stronger evidence, but they indicated no statistical difference in pacifier use between children with and those without ECC. Furthermore, the reported odds ratios suggested that pacifier use might have had a mildly protective effect.

Conclusions The evidence does not suggest a strong or consistent association between pacifier use and ECC. More studies (with either a cohort or a case-control design) that control for other relevant variables and that clearly investigate the role of the pacifier alone must be conducted before a clear recommendations can be made.

\section{Commentary}

This paper is a systematic review which aimed to investigate the relationship, if any, between pacifier use and ECC. Subsidiary aims were to look at the effect of sweetening of the pacifier and duration of use. Unfortunately there is not complete agreement on the definition of ECC and the papers included suffer from this problem. The author excluded studies that reported caries in general, rather than specifically ECC, and it could be argued that this decision weakened the review, particularly because of the small number of papers available. The definition of a "sweetened comforter" also caused problems because this included various designs, including bottles containing sweetened liquid.

Eight studies were included, none of which were considered to provide strong evidence as assessed by a standard validity tool. There was wide variation in the methodology used, with confounding factors not being controlled for in the majority of studies.

As with so many systematic reviews, this one reports a general lack of evidence. It identified no consistent association between pacifier use and ECC. There was some suggestion that a pacifier may provide some protection against caries. The author does not speculate upon a reason for this but one could imagine that it might be due to an increase in salivation. One of the reviewed papers also suggested a tendency for sweetened pacifiers to be protective. This I find difficult to believe but, depending on sweetening agent used and duration of use of the pacifier, it may be possible. It is, however, more likely to be due to a lack of control for confounding factors.

This appears to have been a well-conducted review. Small points of criticism are that only one person rather than two assessed the papers, therefore increasing the risk of error. Further, only articles in English were included, presumably because of the difficulty in having papers translated. Some indication of the number of studies excluded on the basis of language would have been interesting.

The review makes suggestions on how to improve the quality of future studies and these suggestions should be followed by future researchers.

\section{Practice point}

- There is a clear need to improve the quality of research in order to discover any association between pacifier use and childhood caries.

\author{
Chris Deery \\ Edinburgh Dental Institute, Edinburgh, Scotland, UK
}

Evidence-Based Dentistry (2004) 5, 44.

doi:10.1038/sj.ebd. 6400249 